\title{
Photoluminescence characteristics of InAs self-assembled quantum dots in InGaAs/GaAs quantum well
}

\author{
Lingmin Kong \\ Department of Physics, Zhejiang Ocean University, Zhoushan 316000, China
}

\author{
Zhengyun Wu \\ Department of Physics, Xiamen University, Xiamen 361005, China \\ Zhe Chuan Fenga) \\ Graduate Institute of Electro-Optical Engineering and Department of Electrical Engineering, \\ National Taiwan University, Taipei 106-17, Taiwan, Republic of China \\ Ian T. Ferguson \\ School of Electrical Engineering, Georgia Institute of Technology, Atlanta, Georgia 30332
}

(Received 21 March 2007; accepted 27 April 2007; published online 18 June 2007)

\begin{abstract}
Three different InAs quantum dots (QDs) in an InGaAs/GaAs quantum well were formed and investigated by time-resolved and temperature dependent photoluminescence (PL). A strong PL signal emitting at $\sim 1.3 \mu \mathrm{m}$ can be obtained at room temperature with a full width at half maximum of only $28 \mathrm{meV}$. Dots-in-a-well structures result in strong stress release and large size InAs QDs which lead to narrowing and redshifting of PL emissions, enhancement of carrier migration, increasing carrier density in QDs, achievement of good PL lifetime stability on temperature, and improving the QD quality. (C) 2007 American Institute of Physics. [DOI: 10.1063/1.2745410]
\end{abstract}

Semiconductor quantum dots (QDs) are of great interest in both fundamental and technological points of view, due to their unique physical and optoelectronic properties, in particular, the strong three-dimensional (3D) confinement of carriers in nanometer scale QDs. ${ }^{1,2}$ The self-assembled semiconductor QDs, formed by utilizing the lattice mismatch between the substrate and deposited materials, have been attracting great attention. ${ }^{3}$ Strain is the driving force of this growth mode, known as Stranski-Krastanow (SK) mode, which causes the transition from two-dimensional (2D) growth to the formation of defect-free islands. ${ }^{1,4}$ Various QD structures have been prepared by the SK technique, among which InAs/GaAs QD structure is an attractive one because both InAs and GaAs are direct-gap semiconductor and it can be easily prepared for photoconducting devices.

Recently, a type of InAs QDs structure has been reported, which is growing the InAs QDs in an InGaAs quantum well $(\mathrm{QW})$, i.e., a so-called dots-in-a-well (DWELL) structure. ${ }^{5,6}$ This DWELL structure can be formatted by enclosing the QDs first with an InGaAs layer followed by a GaAs layer. The DWELL structure can increase the QD density, the QD size, and capture ability to carriers, leading to an improvement of the laser performance. Theoretical calculation indicated that the larger QD sizes or QW width may obtain longer wavelength photoluminescence (PL) emissions. ${ }^{7}$ Although a few exciting experimental and theoretical progresses have been achieved, ${ }^{5,6}$ some basic issues, for example, the dependences of carriers migration on temperature $(T)$, strained relaxed layers, and QD density, have not been well understood. It is believed that the growth of

\footnotetext{
${ }^{\text {a) }}$ Author to whom correspondence should be addressed; Tel: +886-2-33663543; FAX: +886-2-2367-7467; electronic mail: zcfeng@cc.ee.ntu.edu.tw
}

QDs in an InGaAs/GaAs quantum well can give an insight into the physics of self-organization. In this paper, we present a penetrating investigation on the QD emission dynamics via $T$-dependent and time-resolved photoluminescence (TRPL) on InAs QDs in InGaAs/GaAs QWs. Some related phenomena are discussed.

Experimental InAs/GaAs QD samples were grown on GaAs (100) semi-insulating substrates by solid source molecular beam epitaxy (MBE). For samples A and B, with the schematic diagram shown in Fig. 1(a), after a thick GaAs buffer $(500 \mathrm{~nm})$, a $20 \mathrm{~nm}$ GaAs wall layer, and a $5 \mathrm{~nm}$ $\mathrm{In}_{0.2} \mathrm{Ga}_{0.8} \mathrm{As}$ layer were grown and followed, InAs layers of 2.5 ML (monolayer) for sample A and 3.5 ML for sample B were grown to form a random distribution of QDs. Then, the self-assembled QD layer was capped by a $3 \mathrm{~nm} \operatorname{In}_{0.2} \mathrm{Ga}_{0.8} \mathrm{As}$ layer. The DWELL structures consisted of three periods, finally capped with a $60 \mathrm{~nm} \mathrm{GaAs}$ cap layer.

For comparison, a third sample (sample C) was prepared with the same structure as sample B except that sample C
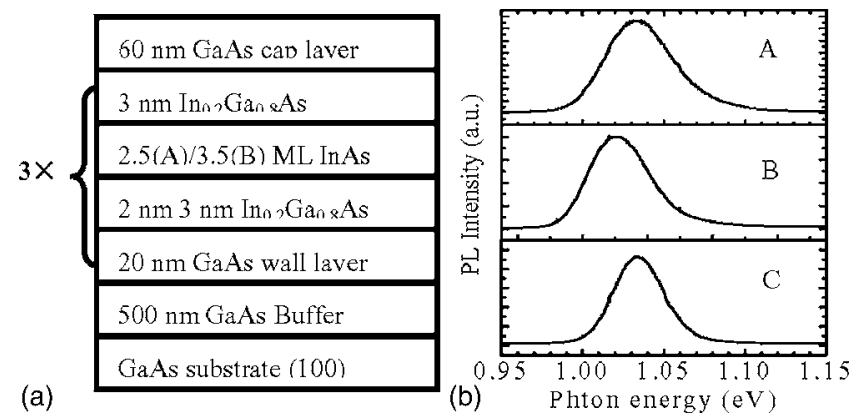

FIG. 1. (a) The schematic diagram for samples A and B. (b) PL spectra of three InAs QD samples at $14 \mathrm{~K}$. Each band can be fitted with two Gaussians. 


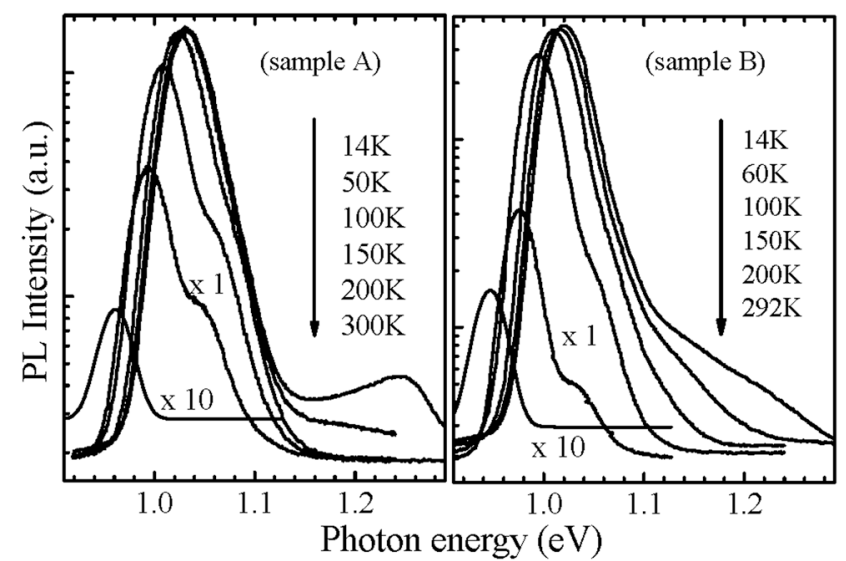

FIG. 2. Temperature dependent PL spectra for samples A and B.

had a single-layer structure. In addition, two extra corresponding samples without GaAs cap were prepared for scanning electron microscopy (SEM) measurements, from which the average dot densities of about $1.8 \times 10^{9} \mathrm{~cm}^{-2}$ for sample A and $2 \times 10^{9} \mathrm{~cm}^{-2}$ for sample B were obtained. Compared with sample A, sample B has larger QD size and higher QD density. These two samples also contain small sizes of QDs.

PL and TRPL were measured in temperature $(T)$ of 14-300 K. A time-correlated single photon counting system was used with a $638 \mathrm{~nm}$ semiconductor laser delivering 100 ps pulses at $20 \mathrm{MHz}$ and a Hamamatsu R5509-72 near infrared (NIR) photomultiplier tube (PMT).

Figure 1(b) shows PL spectra of three DWELL samples measured at $14 \mathrm{~K}$. Fitted by two Gaussians, the full width at half maximum (FWHM) values for the main peak of three samples $\mathrm{A}, \mathrm{B}$, and $\mathrm{C}$ are $36.8,34.2$, and $30.0 \mathrm{meV}$, respectively. These FWHM values are small in comparison with recent results, ${ }^{8}$ indicating the high growth quality of our QD samples. At $14 \mathrm{~K}$, the PL peak energy of sample B is $1.0208 \mathrm{eV}$, which is smaller than those of samples $\mathrm{A}$ $(1.0320 \mathrm{eV})$ and $\mathrm{C}(1.0344 \mathrm{eV})$. The PL peak energy is very dependent on growth conditions. A redshift of PL peak is observed from samples A and C to sample B, which can be attributed to the multilayer growth and an increase of InAs layer thickness. Sufficient stress release for the upper layer QDs can appear within the multilayer QD structure, leading to a decrease of the QD emission energy. ${ }^{9}$ This also causes the broadening of QD PL emissions, ${ }^{9}$ leading to a larger PL FWHM of samples A and B than sample C. Further, larger QD size due to an increase of InAs QD layer thickness may also generate longer wavelength PL emissions.

Figure 2 exhibits $T$-dependent PL spectra for samples A and $\mathrm{B}$ in 14-300 K (some shown only). An obvious PL redshift and a reduction in PL intensity can be observed as $T$ increases. It is seen that PL spectra at every $T$ can be fitted by two Gaussians. All PL spectra in Fig. 2 consist of two peaks, labeled by peak 1 for the lower energy and peak 2 for the higher one. Peak 1 is the main PL peak for all cases, due to emissions from QDs. Peak 2 has a weaker emission, related to the InGaAs strain layer in the DWELL structure, whereas dots grown on GaAs and covered with InGaAs present a more symmetric PL line shape. ${ }^{10}$

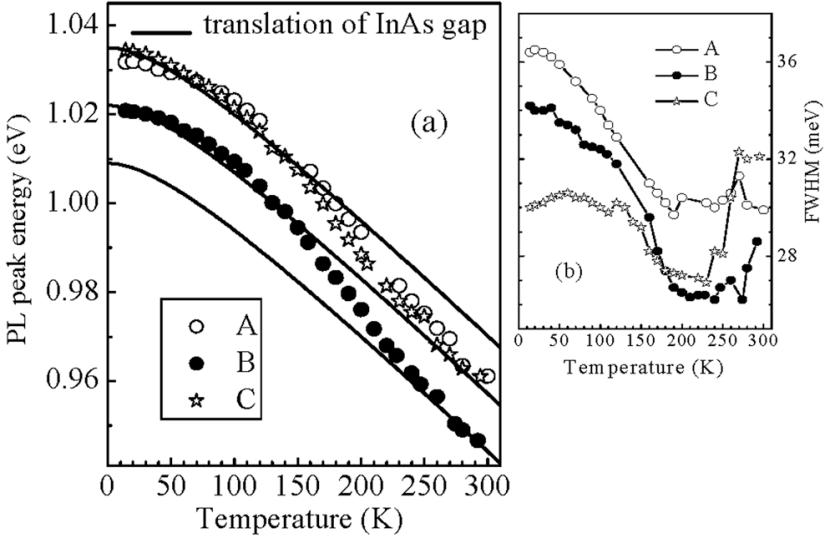

FIG. 3. PL energy and FWHM of the main peak vs temperature.

Different from single-layer InAs QDs, for the equal thickness of every period, the strain transition effect of multilayer structure induces an effect to influence the size of self-aligned QDs, having the QDs at the upper layer with larger sizes. In addition, there exist some no-self-aligned QDs usually with smaller sizes. These two factors cause a distribution of QD sizes, formatting both larger and smaller size QDs. From the self-aligned effect of larger size QDs, the number of larger size QDs is much more than smaller ones and the PL intensity of larger size QDs is much stronger.

The emission wavelength from the $\operatorname{In}(\mathrm{Ga}) \mathrm{As}$ QDs on GaAs is typically around $1.0 \mu \mathrm{m}$ at room temperature (RT). ${ }^{11}$ PL peak wavelengths of our three DWELL samples at RT are around $1.3 \mu \mathrm{m}$, which are longer than that of common QDs directly grown on GaAs matrix, and more suitable for optical fiber communication applications. This is due to the DWELL structure with InAs/InGaAs QDs in a QW. The lattice constant of InGaAs is very close to that of InAs, which may result in stress release among InAs QDs, leading to the formation of large InAs QDs. It can narrow the band gap of InAs QDs and lead to a redshift of PL emissions. In addition, the energy sublevel overlap between quantum wells and dots may increase the carrier density in QDs, ${ }^{12}$ and it is experimentally observed that multilayer structures with DWELL can greatly increase the QD PL intensity. ${ }^{5}$

Figure 3 exhibits the relationships of PL energy and FWHM of the main peak with temperature. All three DWELL samples show an anomalous redshift of PL peak energy with increasing $T$ within $14-300 \mathrm{~K}$. At low $T$ $(<150 \mathrm{~K})$, sample $\mathrm{C}$ has the least FWHM value, and sample A has the largest one. However, FWHM values of samples A and B gradually decrease as $T>150 \mathrm{~K}$. Finally, the FWHM of sample B reaches the smallest one of $26.2 \mathrm{meV}$ at $\sim 240 \mathrm{~K}$, and on the contrary, that of sample $\mathrm{C}$ comes to the largest one at $T$ of $\sim 270 \mathrm{~K}$. These reveal a fact that in the multilayer DWELL structure, the carrier migration intensifies with $T$ among different QD layers through the thin wall layer. In contrast, the migration is very weak when the wall layer is up to $50 \mathrm{~nm}$, which results in a little change of PL FWHM with $T$. Therefore, these results indicate the enhancement of carrier migration among different QDs of DWELL samples in this $T$ range. 


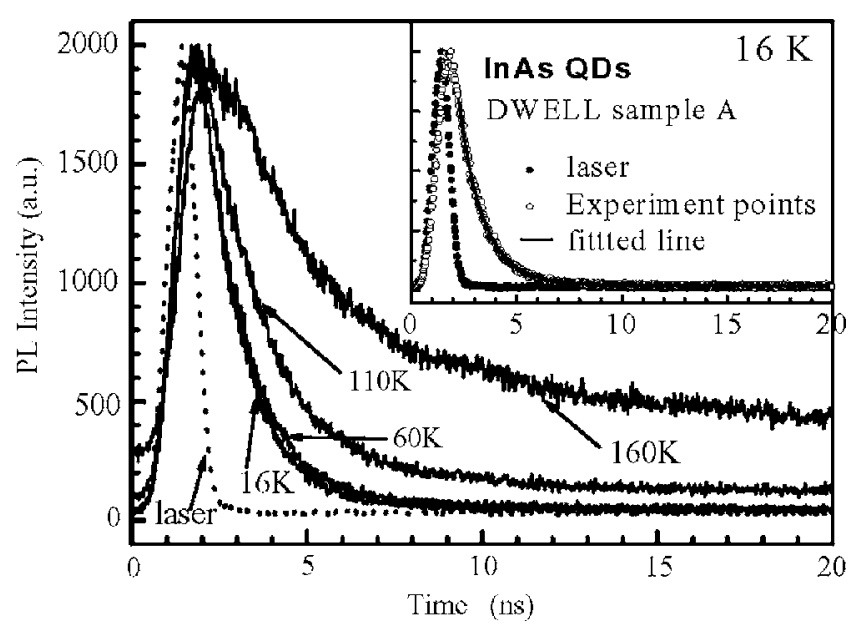

FIG. 4. Time-resolved PL spectra at several $T$ for DWELL sample A and decay curve fitting (insert) for a TRPL spectrum measured at $16 \mathrm{~K}$.

TRPL was measured for three InAs QD DWELL samples. Figure 4 shows TRPL spectra of sample A at several typical $T$ and a typical decay curve fitting with a single exponential process for TRPL at $16 \mathrm{~K}$. Figure 5 exhibits the relationship of PL peak lifetime versus $T$ for three DWELL samples.

It is seen that at low $T(<50 \mathrm{~K})$, the PL peak lifetime varies little with $T$, indicating the weak carrier migration among different QDs, which is same as PL characteristics of a single QD. PL lifetimes of all three samples increase as $T$ rises from 50 to $\sim 150 \mathrm{~K}$, which can be attributed to different mechanisms such as the thermally induced population of dark excitons and thermally induced carrier redistribution among QDs. We consider that the rate of carriers from GaAs or InGaAs QW layer to QDs decreases for scattering as $T$ rises, which favors the increase of PL lifetime. As $T$ moves beyond $50 \mathrm{~K}$, the overall variation of PL peak lifetime from both samples $\mathrm{B}$ and $\mathrm{C}$ is larger than that of sample $\mathrm{A}$, which may be related to the increase of density and size of QDs due to an increase of the InAs layer thickness. For larger QD diameters, electron and hole wave functions expand in different directions and become more elongated, hence reducing the overlap wave function and resulting in a longer lifetime. Multi-QW (MQW) structures may increase QD exciton

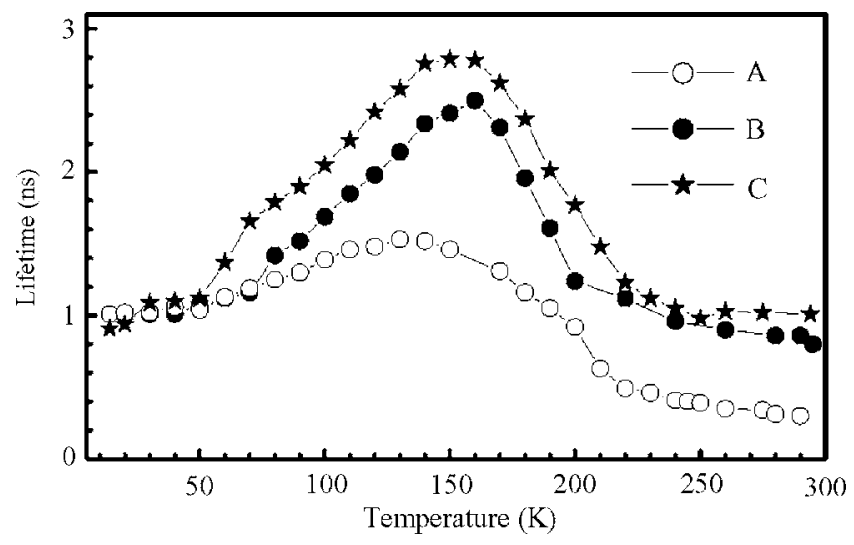

FIG. 5. Lifetime vs $T$ for three DWELL samples A, B, and C. oscillator strength and decrease radiative lifetime, so sample $\mathrm{C}$ has a larger PL peak lifetime than samples A and B.

A significant variation appears at $\sim 150 \mathrm{~K}$, beyond which PL lifetime decreases (until RT). It can casually be attributed to the thermal activation of nonradiative processes. Compared with QDs directly grown on GaAs matrix or InGaAs strained layer, the InAs QDs grown in an InGaAs quantum well exhibit a slight overall variation of PL lifetime (1.2, 1.7, and $1.9 \mathrm{~ns}$ for samples A, B, and C, respectively), corresponding to PL characteristics, which shows that the DWELL structures have a better stability on temperature than the QDs not grown inside a QW.

In summary, we have shown that by using the structure of InAs QDs in an InGaAs/GaAs quantum well on GaAs substrate, long wavelength emission near $1.3 \mu \mathrm{m}$ can be achieved. Our results predicte that the close lattice constant of InGaAs to that of InAs may result in stress release among InAs QDs and large sizes of InAs QDs, which greatly improves the QD quality, narrows the band gap of InAs QDs, and leads to the redshift of PL emissions. In addition, the energy sublevel overlap between quantum wells and dots may increase the carrier density in QDs. It is found that in the multilayer DWELL structure, the carrier migration intensifies with temperature among different QD layers through the thin wall layer, resulting in a large decrease of PL FWHM with $T$. Compared with the InAs QDs directly grown on GaAs matrix or InGaAs strained layer with InAs QDs, the InAs QDs grown in an InGaAs quantum well exhibit a slight overall variation of PL lifetime. Our results provide good evidence of the stability on temperature from DWELL structures.

We acknowledge the support of Dr. Zhichuan Niu and help from Nola Li. This work was supported partially by the Science and Technology Project of Zhoushan City, Grant No. 06110, and National Taiwan University by funds of NSC 94-2215-E-002-019 and NSC 95-2221-E-002-118.

${ }^{1}$ J. Ibáñez et al., J. Appl. Phys. 99, 043501 (2006).

${ }^{2}$ J. H. Lee, Z. M. Wang, B. L. Liang, K. A. Sablon, N. W. Strom, and G. L. Salamo, Semicond. Sci. Technol. 21, 1547 (2006).

${ }^{3}$ N. K. Cho, S. P. Ryu, J. D. Song, W. J. Choi, J. I. Lee, and H. Jeon, Appl. Phys. Lett. 88, 133104 (2006).

${ }^{4}$ D. Bimberg, M. Grundmann, and N. N. Ledentsov, Quantum Dot Heterostructures (Wiley, New York, 1999).

${ }^{5}$ G. T. Liu, A. Stintz, H. Li, T. C. Newell, A. L. Gray, P. M. Varangis, K. J. Malloy, and L. F. Lester, IEEE J. Quantum Electron. 36, 1272 (2000).

${ }^{6}$ A. I. Tartakovskii, R. S. Kolodka, H. Y. Liu, M. A. Migliorato, M. Hopkinson, M. N. Makhonin, D. J. Mowbray, and M. S. Skolnick, Appl. Phys. Lett. 88, 131115 (2006).

${ }^{7}$ M. A. Cusack, P. R. Briddon, and M. Jaros, Phys. Rev. B 54, R2300 (1996).

${ }^{8}$ Yu. I. Mazur, B. L. Liang, Zh. M. Wang, D. Guzun, G. J. Salamo, Z. Ya. Zhuchenko, and G. G. Tarasov, Appl. Phys. Lett. 89, 151914 (2006).

${ }^{9}$ H. Saito, K. Nishi, and S. Sugou, Appl. Phys. Lett. 73, 2742 (1998).

${ }^{10}$ A. Fiore, P. Borri, W. Langbein, J. M. Hvam, U. Oesterle, R. Houdré, R. P. Stanley, and M. Ilegems, Appl. Phys. Lett. 76, 3430 (2000).

${ }^{11}$ C. Annalisa, C. Luciana, L. Gavriella, and V. Sesto, J. Cryst. Growth 261, 458 (2004).

${ }^{12}$ F. Pulizzi, A. J. Kent, A. Patanè, L. Eaves, and M. Henini, Appl. Phys. Lett. 84, 3046 (2004). 